Journal of Applied Pharmaceutical Science Vol. 6 (12), pp. 197-201, December, 2016

Available online at http://www.japsonline.com

DOI: $10.7324 /$ JAPS.2016.601229

ISSN 2231-3354 (cc) BY-NC-SA

\title{
Triterpenes from Pouteria gardneri (Mart. \& Miq.) Baehni extracts
}

\author{
Cintia Alves de Matos Silva ${ }^{1}$, Ricardo de Oliveira e Melo ${ }^{1}$, Inês Sabioni Resck ${ }^{2}$, Dâmaris Silveira ${ }^{1 *}$ \\ ${ }^{1}$ Faculdade de Ciências da Saúde, Universidade de Brasília, Campus Universitário Darcy Ribeiro, Asa Norte, Brasília, DF, Brazil. \\ ${ }^{2}$ Instituto de Química. Universidade de Brasilia, Campus Universitário Darcy Ribeiro, Asa Norte, Brasília, DF, Brazil.
}

\begin{tabular}{l} 
ARTICLE INFO \\
\hline Article history: \\
Received on: $24 / 07 / 2016$ \\
Revised on: $16 / 08 / 2016$ \\
Accepted on: $13 / 09 / 2016$ \\
Available online: $28 / 12 / 2016$ \\
\hline Key words: \\
Pouteria gardneri, \\
Sapotaceae, Cerrado, \\
medicinal plant.
\end{tabular}

\section{INTRODUCTION}

Sapotaceae is a pantropical family of flowering plants (Alves-Araújo and Alves, 2013). In Brazil, there are 12 genera and about 230 known species (Carneiro et al., 2015). Pouteria is the largest genus of Sapotaceae (Alves-Araújo et al., 2014). Although 122 species had been recorded in Brazil (Alves-Araujo, 2015), only a few species were evaluated for the chemistry composition. It was observed that triterpene and steroidal compounds mean to be quite common in this genus (Anjaneyulu, 1965; Ardon and Nakano, 1973; Che et al., 1980; Pellicciari et al., 1972; Perfeito et al., 2005; Silva et al., 2009). From polyphenol compounds, myricitrin is, usually, found in Pouteria species and maybe can be considered a Pouteria chemical marker (Lim, 2013; Ma et al., 2003; Ma et al., 2004). Pouteria gardneri (Mart. \& Miq.) Baehni is one of the Brazilian native species, and is commonly known as "cabritão", "frutinha-de-veado",

\footnotetext{
* Corresponding Author

Damaris Silveira, Faculdade de Ciências da Saúde, Universidade de Brasília, Campus Universitário Darcy Ribeiro, Asa Norte, Brasília, DF, Brazil. Phone: +55 61 31071939,Email: damaris@unb.br
}

"sapotinha", "leiteiro-preto", or "leiteiro-da-folha-miuda" (AlvesAraújo and Alves, 2013; Coelho et al., 2009a; Mesquita et al., 2005). This species can be found in almost all Brazilian biomes, including Cerrado (Alves-Araujo et al., 2016). Also, P. gardneri can be found in Bolivia and Paraguay (TROPICOS, 2014). The wood is used to build houses, and the small fruits are eaten freshly (Paula et al., 2011). Extracts from P. gardneri were evaluated about trypanocidal, antimalarial and anti-leishmania activities and did not present activity (Mesquita et al., 2005; Mesquita et al., 2007). The same researchers evaluated $P$. gardneri extract about cytotoxicity against four cell lines (SF-295, HCT-8, MDA-MB435, and HL-60) and no activity was found (Mesquita et al., 2009).

Also, several extracts from this species were tested for toxicity to Dipetalogaster maxima and Aedes aegypti larvae and did not present significant activity (Coelho et al., 2009a; Coelho et al., 2009b). Extracts from leaves of $P$. gardneri also were evaluated for potential enzyme inhibition. The extracts did not show inhibition of tyrosinase (Souza et al., 2012a). However, the ethanol and hexane crude extracts presented $\alpha$-glucosidase $(100$ and $95 \%$, respectively) and $\alpha$-amylase (71 and $31 \%$, respectively) inhibition (Souza et al., 2012b). 
Therefore, because there is a lack of knowledge about chemical composition P. gardneri, the aim of this work is to report the phytochemical study of hexane and ethanol extracts from leaves.

\section{MATERIAL AND METHODS}

All process was monitored by analytical TLC, performed on precoated ALUGRAM sil G Machery-Nagel silica gel (60/0.2 $\mathrm{mm}$ ) plates, using anisaldehyde reagent (Wagner and Bladt, 1996). ${ }^{1} \mathrm{H}$ and ${ }^{13} \mathrm{C}$ NMR spectra were registered on Varian $(7.05 \mathrm{~T})$ Mercury-plus spectrometers, operating at $300 \mathrm{MHz}$ and performed in $\mathrm{CDCl}_{3}$. Offline processing was conducted using Mestre-C NMR Software (Mestrelab Research).

Analytical GC-MS was carried out on a Thermo Finnigan system with chromatograph TRACE GC coupled to mass spectrometer Polaris Q (EI, $70 \mathrm{eV}$ ) fitted with HP5MS (5\% diphenyl, $95 \%$ dimethylpolysiloxane) capillary column (30 $\mathrm{m} \times 0.25 \mathrm{~mm} \times 0.25 \mathrm{~mm})$. The carrier gas was helium with flow rate $0.6 \mathrm{~mL} / \mathrm{min}$. The temperature of the column was programmed from $60{ }^{\circ} \mathrm{C}(1 \mathrm{~min})-40{ }^{\circ} \mathrm{C} / \mathrm{min}$ to $140{ }^{\circ} \mathrm{C}$ and $4{ }^{\circ} \mathrm{C} / \mathrm{min}$ to $300{ }^{\circ} \mathrm{C}$ (10 min). The injection volume was $0.2 \mu \mathrm{L}$ solution $1 \mathrm{mg} / \mathrm{mL}$ (sample in chloroform). The obtained mass spectra were compared to NIST standard reference database.

\section{Plant material}

The leaves of Pouteria gardneri were collected at Paranoá - DF, in July 2003 and identified by Professor J. E. de Paula. An exsiccate was deposited at Herbarium of Universidade de Brasília (UB) (voucher number JElias de Paula 3718). The extraction was performed according to previously reported technique (Perfeito et al., 2005).

Briefly, the dried and powdered plant material (141.8 g) was macerated at room temperature for seven days (repeated three times) with hexane. After filtration, the solvent from the extractive solution was eliminated under reduced pressure, at $40{ }^{\circ} \mathrm{C}$, furnishing $5.3 \mathrm{~g}$ (3.7\% yield) of crude hexane extract (HG) and $22.9 \mathrm{~g}$ (16.2\% yield) of crude ethanol extract (EG).

\section{Isolation procedures}

Hexane extract HG $(3.0 \mathrm{~g})$ was submitted to filtration over Silica gel 60 Merck, furnishing 5 fractions according to the used eluent: hexane (HGA, $300.0 \mathrm{mg})$, hexane/ethyl acetate (1:1) (HGB, $2.4 \mathrm{~g}$ ), ethyl acetate (HGC, $100.0 \mathrm{mg}$ ), ethyl acetate/ methanol (1:1) (HGD, $100.0 \mathrm{mg}$ ) and methanol (HGE, $0.3 \mathrm{mg}$ ). HGB fraction (1.5 g), after column chromatography over silica (Silica gel 60 Merck), using hexane- AcOEt- MeOH gradient, furnished 17 groups of fractions. Group HGB3 (92.9 mg), eluted with Hex: AcOEt (9:1) was a mixture of triterpenes (1) and longchain fatty esters. Further silica gel column chromatography of HGB3 led to the isolation of (2) (22 mg).

Ethanol extract EG (3.2 g) was partitioned using Hexane: $\mathrm{CHCl}_{3}: \mathrm{CH}_{3} \mathrm{CN}: \mathrm{H}_{2} \mathrm{O}$ (2:1: 3.4:1), as previously described (Perfeito et al., 2005). The process led to three fractions: hexane
(EGF, $37 \mathrm{mg}$ ) $\mathrm{CHCl}_{3}: \mathrm{CH}_{3} \mathrm{CN}$ (EGG, $0.5 \mathrm{~g}$ ) and aqueous (EGH, $2.6 \mathrm{~g})$. Part of $\mathrm{CHCl}_{3}: \mathrm{CH}_{3} \mathrm{CN}$ fraction EGG $(0.1 \mathrm{~g})$ was submitted to silica gel column using hexane - ethyl acetate gradient. The fractions presenting similar TLC profile were pooled, furnishing 18 groups. Group 3 (eluted by Hex: AcOEt (9:2) gave (3).

\section{RESULTS}

The obtained compounds were characterized by ${ }^{1} \mathrm{H}$, and ${ }^{13} \mathrm{C}$ NMR spectra, by comparison with literature data (Mahato and Kundu, 1994; Pellicciari et al., 1972; Sholichin et al., 1980). The analysis of ${ }^{13} \mathrm{C}$ NMR of $(\boldsymbol{1})$ allowed the detection of signals corresponding to acetate of $\alpha^{-}$and $\beta$-amyrin and lupeol (Table 1).

Table 1: Chemical shifts of $1\left(\delta, \mathrm{CDCl}_{3}, 75 \mathrm{MHz}\right)$ in comparison with literature data $\left(\delta, \mathrm{CDCl}_{3}, 75 \mathrm{MHz}\right)$ (Mahato and Kundu, 1994; Sholichin et al., 1980).

\begin{tabular}{|c|c|c|c|c|c|}
\hline C & $1(\delta)$ & $\begin{array}{c}\text { Lupeol } \\
\text { acetate }^{1}\end{array}$ & $1(\delta)$ & $\begin{array}{c}\alpha- \\
\text { amyrin }^{2}\end{array}$ & $\begin{array}{c}\beta- \\
\text { amyrin }^{2}\end{array}$ \\
\hline 1 & 38.4 & 38.4 & 38.4 & 38.7 & 38.7 \\
\hline 2 & 23.7 & 23.7 & 27.4 & 27.2 & 27.3 \\
\hline 3 & 80.9 & 81.0 & 80.9 & 78.3 & 79.0 \\
\hline 4 & 37.8 & 37.8 & 38.4 & 38.7 & 38.8 \\
\hline 5 & 55.3 & 55.4 & $55.2 ; 55.3$ & 55.2 & 55.3 \\
\hline 6 & 18.2 & 18.2 & 18.2 & 18.3 & 18.5 \\
\hline 7 & 34.3 & 34.3 & 32.8 & 32.9 & 32.8 \\
\hline 8 & 40.8 & 40.9 & 39.9 & 40.0 & 38.8 \\
\hline 9 & 50.3 & 50.4 & 47.6 & 47.7 & 47.7 \\
\hline 10 & 37.1 & 37.1 & $36.9 ; 37.6$ & 36.9 & 37.6 \\
\hline 11 & 20.9 & 21.0 & $23.3 ; 23.6$ & 23.3 & 23.6 \\
\hline 12 & 25.0 & 25.1 & $124.3 ; 121.6$ & 124.3 & 121.8 \\
\hline 13 & 38.0 & 38.1 & $139.6 ; 145.1$ & 139.3 & 145.1 \\
\hline 14 & 42.9 & 42.9 & $42.0 ; 41.7$ & 42.0 & 41.8 \\
\hline 15 & 27.4 & 27.5 & $28.7 ; 26.2$ & 28.7 & 26.2 \\
\hline 16 & 35.5 & 35.6 & $26.6 ; 26.9$ & 26.6 & 27.0 \\
\hline 17 & 43.0 & 43.0 & $33.7 ; 32.5$ & 33.7 & 32.5 \\
\hline 18 & 48.2 & 48.3 & $59.0 ; 47.5$ & 58.9 & 47.4 \\
\hline 19 & 48.0 & 48.0 & $39.6 ; 46.7$ & 39.6 & 46.9 \\
\hline 20 & 150.9 & 150.9 & $39.6 ; 31.0$ & 39.6 & 31.1 \\
\hline 21 & 29.8 & 29.9 & $31.2 ; 34.7$ & 31.2 & 34.8 \\
\hline 22 & 40.0 & 40.0 & $41.5 ; 37.1$ & 41.5 & 37.2 \\
\hline 23 & 28.0 & 28.0 & 28.0 & 28.1 & 28.2 \\
\hline 24 & 16.5 & 16.5 & 15.5 & 15.6 & 15.5 \\
\hline 25 & 16.1 & 16.2 & 15.7 & 15.6 & 15.6 \\
\hline 26 & 16.0 & 16.0 & $16.7 ; 16.8$ & 16.8 & 16.9 \\
\hline 27 & 14.5 & 14.5 & $23.3 ; 25.9$ & 23.3 & 26.0 \\
\hline 28 & 18.0 & 18.0 & $28.0 ; 28.4$ & 28.1 & 28.4 \\
\hline 29 & 109.3 & 109.4 & $17.4 ; 33.3$ & 17.4 & 33.3 \\
\hline 30 & 19.2 & 19.3 & $21.3 ; 23.7$ & 21.3 & 23.7 \\
\hline $\mathrm{CH}_{3} \mathrm{CO}$ & 21.3 & 21.3 & - & - & - \\
\hline $\mathrm{CH}_{3} \mathrm{CO}$ & 171.0 & 170.8 & - & - & - \\
\hline
\end{tabular}

To confirm the presence of acetate of $\alpha$ - and $\beta$ - amyrin and lupeol in (1), a GC-MS analysis was carried out. Based on fragmentation patterns, the analysis showed (1), besides the identified compounds, also presented long fatty esters, $\alpha$-amyrin acetate and $\psi$-taraxasterol acetate (Table 2). The column chromatography of ( $\boldsymbol{1})$, led to the isolation of $\alpha$-amyrin acetate (2) (Table 3). The ${ }^{13} \mathrm{C}$ NMR spectrum of (3) also shows the presence of a mixture of two triterpenes. The comparison with literature data led to suggest the presence of ursolic and oleanolic acids (Table 4). 
Table 2: Detected triterpenes in hexane extract of Pouteria gardneri leaves by GC-MS analysis.

\begin{tabular}{clcc}
\hline RT $(\mathbf{m i n})$ & \multicolumn{1}{c}{ Compound } & mass & Main observed fragmentation $(\mathbf{m} / \mathbf{z})$ \\
\hline 48.89 & $\beta$-amyrin & 468 & $426(\mathrm{M}+) ; 411 ; 408 ; 229 ; 218 ; 205 ; 189 ; 109(100 \%) ; 95$ \\
49.19 & $\alpha$-amyrin acetate & 468 & $468(\mathrm{M}) ; 408 ; 365 ; 249 ; 218(100 \%) ; 203 ; 189$ \\
49.36 & $\psi$-taraxasterol acetate & 468 & $468(\mathrm{M}+) ; 453 ; 218 ; 189(100)$ \\
50.26 & $\beta \square$ amyrin acetate & 468 & $468(\mathrm{M}+) ; 453 ; 218(100) ; 203 ; 189$ \\
50.45 & lupeol acetate & 468 & $468(\mathrm{M}+) ; 453 ; 408 ; 189(100)$ \\
\hline
\end{tabular}

Table 3: Chemical shifts of $2\left(\delta, \mathrm{CDCl}_{3}, 75 \mathrm{MHz}\right)$ in comparison with literature data $\left(\delta, \mathrm{CDCl}_{3}, 75 \mathrm{MHz}\right)(\mathrm{Ebajo} \mathrm{Jr}$ et al., 2015).

\begin{tabular}{|c|c|c|}
\hline $\mathbf{C}$ & $2(\beta)$ & $\alpha$-amyrin acetate \\
\hline 1 & 38,4 & 38,4 \\
\hline 2 & 27,9 & 27,6 \\
\hline 2 & 28,0 & 28,0 \\
\hline 3 & 80,6 & 80,9 \\
\hline 4 & 38,0 & 37,7 \\
\hline 5 & 55,2 & 55,2 \\
\hline 6 & 18,2 & 18,2 \\
\hline 7 & 32,8 & 32,8 \\
\hline 8 & 40,0 & 40,0 \\
\hline 9 & 47,5 & 47,6 \\
\hline 10 & 36,7 & 36,8 \\
\hline 11 & 23,3 & 23,4 \\
\hline 12 & 124,3 & 124,3 \\
\hline 13 & 139,6 & 139,6 \\
\hline 14 & 42,0 & 42,05 \\
\hline 15 & 26,9 & 26,6 \\
\hline 16 & 28,0 & 28,1 \\
\hline 17 & 33,7 & 33,7 \\
\hline 18 & 59,0 & 59,0 \\
\hline 19 & 39,6 & 39,6 \\
\hline 20 & 39,6 & 39,6 \\
\hline 21 & 31,2 & 31,2 \\
\hline 22 & 41,5 & 41,5 \\
\hline 23 & 28,7 & 28,7 \\
\hline 24 & 16,8 & 16,8 \\
\hline 25 & 15,7 & 15,7 \\
\hline 25 & 16,1 & 16,1 \\
\hline 26 & 16,8 & 16,8 \\
\hline 27 & 23,2 & 23,2 \\
\hline 28 & 28,7 & 28,7 \\
\hline 29 & 17,4 & 17,5 \\
\hline 30 & 21,3 & 21,4 \\
\hline $\mathrm{CH}_{3} \mathrm{CO}$ & 171,0 & 170,0 \\
\hline $\mathrm{CH}_{3} \mathrm{CO}$ & 21,3 & 21,3 \\
\hline
\end{tabular}<smiles>CC(=O)OC1CCC2(C)C(CCC3=C4CC(C)(C)CCC4(C)CCC32C)C1(C)C</smiles>

$\delta$-amyrin acetate<smiles>CC(=O)OC1CCC2(C)C(CCC3(C)C2CC=C2C4C(C)C(C)CCC4(C)CCC23C)C1(C)C</smiles><smiles>CC(=O)OC1CCC2(C)C(CCC3(C)C2CC=C2C4CC(C)(C)CCC4(C)CCC23C)C1(C)C</smiles>

$\alpha$-amyrin acetate (2) $\beta$-amyrin acetate<smiles>CC(=O)OC1CCC2(C)C3CCC4(C)CCC5(C)CC=C(C)C(C)C5C4(C)C3CCC2C1(C)C</smiles>

$\psi$-taraxasterol acetate<smiles>C=C(C)C1CCC2(C)CCC3(C)C(CCC4C5(C)CCC(OC(C)=O)C(C)(C)C5CCC43C)C12</smiles>

lupeol acetate 


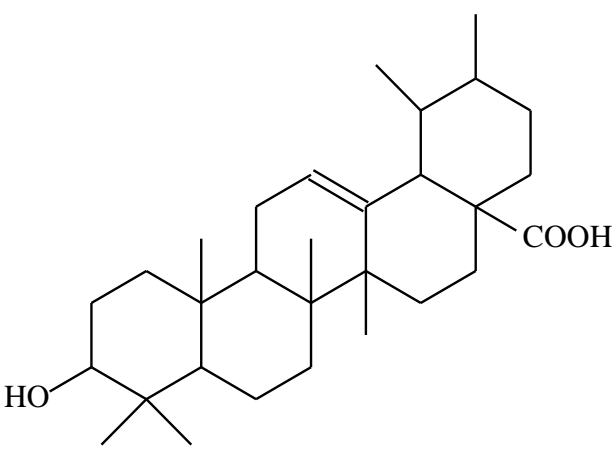

ursolic acid<smiles>CC1(C)CCC2(C(=O)O)CCC3(C)C(=CCC4C5(C)CCC(O)C(C)(C)C5CCC43C)C2C1</smiles>

oleanolic acid

Table 4: Chemical shifts of $3\left(\delta, \mathrm{CDCl}_{3}, 75 \mathrm{MHz}\right)$ in comparison with literature data $\left(\delta, \mathrm{CDCl}_{3}, 50 \mathrm{MHz}\right)$ (Falcão et al., 2003).

\begin{tabular}{|c|c|c|c|c|}
\hline $\mathbf{C}$ & $3(\boldsymbol{\beta})$ & Ursolic acid & $3(\boldsymbol{\beta})$ & Oleanolic acid \\
\hline 1 & 38.7 & 38.8 & 38.5 & 38.5 \\
\hline 2 & 27.1 & 27.3 & 27.1 & 27.4 \\
\hline 3 & 79.0 & 78.8 & 79.0 & 78.7 \\
\hline 4 & 38.7 & 38.8 & 38.7 & 38.7 \\
\hline 5 & 55.2 & 55.4 & 55.2 & 55.2 \\
\hline 6 & 18.2 & 18.4 & 18.2 & 18.3 \\
\hline 7 & 32.9 & 33.0 & 32.6 & 32.6 \\
\hline 8 & 39.4 & 39.6 & 39.4 & 39.3 \\
\hline 9 & 47.5 & 47.5 & 47.6 & 47.6 \\
\hline 10 & 37.0 & 37.0 & 37.0 & 37.0 \\
\hline 11 & 23.3 & 23.3 & 23.2 & 23.1 \\
\hline 12 & 125.8 & 125.5 & 122.6 & 122.1 \\
\hline 13 & 137.8 & 138.0 & 143.5 & 143.4 \\
\hline 14 & 41.9 & 42.0 & 41.6 & 41.6 \\
\hline 15 & 28.1 & 28.2 & 27.6 & 27.7 \\
\hline 16 & 24.1 & 24.3 & 23.3 & 23.4 \\
\hline 17 & 47.9 & 48.1 & 45.8 & 46.6 \\
\hline 18 & 52.6 & 52.8 & 40.9 & 41.3 \\
\hline 19 & 39.0 & 39.1 & 45.8 & 45.8 \\
\hline 20 & 38,8 & 38,8 & 30.6 & 30.6 \\
\hline 21 & 30.6 & 30.7 & 33.7 & 33.8 \\
\hline 22 & 36.6 & 36.7 & 32.4 & 32.3 \\
\hline 23 & 28.10 & 28.2 & 28.1 & 28.1 \\
\hline 24 & 15.5 & 15.5 & 15.4 & 15.6 \\
\hline 25 & 15.6 & 15.7 & 15.3 & 15.3 \\
\hline 26 & 17.0 & 16,9 & 16.9 & 16.8 \\
\hline 27 & 23.6 & 23.6 & 25.9 & 26.0 \\
\hline 28 & 182.2 & 180.0 & 182.5 & 181.0 \\
\hline 29 & 17.0 & 16,9 & 33.0 & 33.1 \\
\hline 30 & 21.1 & 21.2 & 23.6 & 23.6 \\
\hline
\end{tabular}

\section{DISCUSSION}

The compounds from $P$. gardneri are widespread in higher plants and several biological activities are reported to them. $\alpha$ - and $\beta$-Amyrin, as well their derivatives, are quite common in Pouteria species and were previously reported to $P$. caimito and P. tomentosa (Che et al., 1980; Pellicciari et al., 1972). Moreover, $\alpha$ - and $\beta$-amyrin, as well as the compounds $\alpha$-amyrin acetate, and lupeol acetate were isolated from $P$. torta (Che et al., 1980; Perfeito et al., 2005). However, as far we know, it is the first time the chemical study is reported to Pouteria gardneri.

\section{ACKNOWLEDGEMENTS}

The authors are grateful to Coordination of the Higher Education Personnel Improvement (CAPES), National Council of Technological and Scientific Development (CNPQ), University of Brasilia, The Foundation for Scientific and Technological Entrepreneurship (FINATEC), and The Agency for Funding for Research and Projects (FINEP), for financial support.

Conflict of Interests: There are no conflicts of interest. 


\section{REFERENCES}

Alves-Araujo A. 2015. Pouteria, Lista de Espécies da Flora do Brasil Jardim Botânico do Rio de Janeiro, Rio de Janeiro.

Alves-Araújo A, Alves M. Checklist of Sapotaceae in Northeastern Brazil. Check List, 2013; 9(1):059-062.

Alves-Araujo A, Faria AD, Ribeiro JELS, Monteiro MH. 2016.

Pouteria. [ONLINE] Available at: http://floradobrasil.jbrj.gov.br/reflora/floradobrasil/FB14502. [July 22, 2016].

Alves-Araújo A, Swenson U, Alves M. A Taxonomic Survey of Pouteria (Sapotaceae) from the Northern Portion of the Atlantic Rainforest of Brazil. Syst Botany, 2014; 39(3):915-938.

Anjaneyulu B. Chemical investigation of some Indian plants. . Indian J Chem, 1965; 3(5):237-238.

Ardon A, Nakano T. Triterpenes from the bark of Pouteria caimito. . Planta Med, 1973; 23(4):348-352.

Carneiro CE, Alves-Araujo A, Almeida Jr. EB, Terra-Araujo MH. 2015. Sapotaceae ed.^eds. Lista de Espécies da Flora do Brasil (http://floradobrasil.jbrj.gov.br/jabot/floradobrasil/FB217). Rio de Janeiro: Jardim Botânico do Rio de Janeiro

Che CT, Koike K, Cordell GA, Fong HHS, Dobbertein RH. Triterpenes of Pouteria torta (Sapotaceae). Planta Med, 1980; 43(3):420421.

Coelho AA, Paula JE, Espíndola LS. Efeito de extratos de plantas do Cerrado em Dipetalogaster máxima (Uhler)(Hemiptera, Reduviidae). Rev Bras Entomol, 2009a; 53(3):444-451.

Coelho AM, Paula JE, Espindola LS. Larvicidal activity of plant extracts on Aedes Aegypti (L.) Diptera: Culicidae under laboratory conditions. BioAssay, 2009b; 4(3):1-6.

Ebajo Jr VD, Shen C-C, Ragasa CY. Terpenoids and sterols from Hoya multiflora Blume. J Appl Pharm Sci, 2015; 5(4):33-39.

Falcão DQ, Fernandes SBO, Menezes FS. Triterpenos de Hyptis fasciculata Benth. Braz J Pharmacog, 2003; 13(1):81-83.

Lim T. 2013. Pouteria viridis. ed.^eds. Edible Medicinal And Non-Medicinal PlantsSpringer 143-145.

Ma J, Luo XD, Protiva P, Yang H, Ma C, Basile MJ, Weinstein IB, Kennelly EJ. Bioactive novel polyphenols from the fruit of Manilkara zapota (Sapodilla). J Nat Prod, 2003; 66(7):983-986.

Ma J, Yang H, Basile MJ, Kennelly EJ. Analysis of polyphenolic antioxidants from the fruits of three pouteria species by selected ion monitoring liquid chromatography-mass spectrometry. J Agric Food Chem, 2004; 52(19):5873-5878.

Mahato S, Kundu A. ${ }^{13}$ CNMR spectra of pentacyclic triterpenoids - a compilation and some salient features. Phytochemistry, 1994; 37(6):1517-1575.

Mesquita ML, Desrivot J, Bories C, Fournet A, Paula JE, Grellier P, Espindola LS. Antileishmanial and trypanocidal activity of Brazilian Cerrado plants. Mem Inst Oswaldo Cruz, 2005; 100(7):783-787.
Mesquita ML, Grellier P, Mambu L, Paula JE, Espindola LS. In vitro antiplasmodial activity of Brazilian Cerrado plants used as traditional remedies. J Ethnopharmacol, 2007; 110(1):165-170.

Mesquita ML, Paula JE, Pessoa C, Moraes MO, Costa-Lotufo LV, Grougnet R, Michel S, Tillequin F, Espindola LS. Cytotoxic activity of Brazilian Cerrado plants used in traditional medicine against cancer cell lines. J Ethnopharmacol, 2009; 123(3):439-445.

Paula JE, Imaña-Encinas J, Santana OA, Ribeiro GS, Imaña CR. Levantamento florístico e sua distribuição diamétrica da vegetação de um cerrado sensu stricto e de um fragmento de floresta de galeria no ribeirão Dois Irmãos na APA de Cafuringa, DF, Brasil. Biotemas, 2011; 22(3):35-46.

Pellicciari R, Ardon A, Bellavita V. Triterpenes from Pouteria caimito. Planta Med, 1972; 22(2):196-200.

Perfeito JP, Santos MLS, Lopez KSE, Paula JE, Silveira D. Characterization and biological properties of Pouteria torta extracts: a preliminary study. Braz J Pharmacog, 2005; 15(3):183-186.

Sholichin M, Yamasaki K, Kasai R, Tanaka O. 13C Nuclear Magnetic Resonance of Lupane-Type Triterpenes, Lupeol, Betulin and Betulinic Acid. Chem Pharm Bull, 1980; 28(3):1006-1008.

Silva CA, Simeoni LA, Silveira D. Genus Pouteria: chemistry and biological activity. Braz J Pharmacog, 2009; 19(2A):501-509.

Souza PM, Elias ST, Simeoni LA, de Paula JE, Gomes SM, Guerra ENS, Fonseca YM, Silva EC, Silveira Dm, Magalhaes PO. Plants from Brazilian Cerrado with potent tyrosinase inhibitory activity. PloS one, 2012a; 7(11):e48589.

Souza PM, Sales PM, Simeoni LA, Silva EC, Silveira D, Magalhaes Pde O. Inhibitory activity of alpha-amylase and alphaglucosidase by plant extracts from the Brazilian cerrado. Planta Med, 2012b; 78(4):393-399.

TROPICOS (2014). Missoury botanical garden; 2014 [acesso em 07 fevereiro 2014]. Disponível em: http: //www.tropicos.org. Springer- Verlag.

Wagner H, Bladt S. 1996. Plant Drug Analysis. Berlin:

How to cite this article:

Silva CAM, Melo RO, Resck IS, Silveira D. Triterpenes from Pouteria gardneri (Mart. \& Miq.) Baehni extracts. J App Pharm Sci, 2016; 6 (12): 197-201. 